Manuscript for Microbial Ecology: Host Microbe Interactions

\title{
Probiotic dosing of Ruminococcus flavefaciens affects rumen microbiome structure and function in reindeer
}

\author{
Kirsti E. Præsteng • Phillip B. Pope • Isaac K.O. Cann • Roderick I. Mackie • Svein D. \\ Mathiesen • Lars P. Folkow • Vincent G.H. Eijsink • Monica A. Sundset
}

\begin{abstract}
Kirsti E. Præsteng • Lars P. Folkow・Monica A. Sundset (correspondence)
Department of Arctic and Marine Biology, University of Tromsø, Tromsø, Norway

e-mail: $\underline{\text { monica.a.sundset@uit.no }}$
\end{abstract}

Phillip B. Pope • Vincent G.H. Eijsink

Department of Chemistry, Biotechnology and Food Science, Norwegian University of Life Sciences, Ås, Norway

Isaac K.O. Cann • Roderick I. Mackie

Department of Animal Sciences, University of Illinois at Urbana-Champaign, Urbana, USA

Isaac K.O. Cann • Roderick I. Mackie

Institute of Genomic Biology, University of Illinois at Urbana-Champaign, Urbana, USA

Isaac K.O. Cann

Department of Microbiology, University of Illinois at Urbana-Champaign, Urbana, USA

\author{
Roderick I. Mackie \\ Division of Nutritional Sciences, University of Illinois at Urbana-Champaign, Urbana, USA
}

Svein D. Mathiesen

UArctic EALAT Institute at International Centre for Reindeer Husbandry and The Norwegian School of Veterinary Science, Tromsø, Norway 


\begin{abstract}
Highly cellulolytic bacterial species such as Ruminococcus flavefaciens are regarded essential for the microbial breakdown of cellulose in the rumen. We have investigated the effect of ruminal dosing of $R$. flavefaciens strain 8/94-32 during realimentation of starved reindeer (males, $n=3$ ). Microbiome function measured as in situ digestion of cellulose and food pellets (\% DMD; dry matter disappearance) decreased after probiotic dosing. Microbial community analyses (>100,000 16S rDNA gene sequences for 27 samples) demonstrated that ruminal dosing influenced the microbiome structure; reflected by increased phylogenetic distances from background samples (unweighted UniFrac analysis) and reduced species diversity and evenness. Despite the inability to detect strain 8/94-32 post-dosing, the relative abundance of its affiliate family Ruminococcaceae remained consistent throughout the trial whilst a dominant peak in the genus Prevotella and decline in uncharacterized Bacteroidetes (uBacNR) was observed in treatment samples. No clear relationships were observed between the relative abundance of Ruminococcaceae, Prevotella and uBacNR with cellulose DMD, however Prevotella (negative) and uBacNR (positive) exhibited relationships with pellet DMD. These unexpected effects of ruminal dosing of a cellulolytic bacterium on digestibility, are relevant for other studies on rumen manipulation.
\end{abstract}

Keywords: metagenomics; Ruminococcus flavefaciens; reindeer; 454-pyrosequencing 


\section{Introduction}

Reindeer (Rangifer tarandus tarandus) in northern Norway are ruminants herded in a pastoralistic system, that mainly graze on natural pastures from which they select a large variety of graminoids, woody plants, lichens and mosses [21]. They experience large seasonal variation in feed quality and abundance, particularly during winter when snow conditions occasionally result in years with poor grazing condition thus restricting natural plants availability [13]. To reduce starvation, Saami pastoral herders provide supplementary feed such as baled grass silage and hay as well as commercially produced pellet concentrate which has become increasingly common in Saami reindeer husbandry [31]. Supplementary feeding of reindeer reduces starvation, but digestion problems still occurs and is putatively inferred to the condition of the animals and their ability to digest and utilise the food provided [14, 22].

In the reindeer rumen functions the resident microbiome contributes to the deconstruction of fibrous feed. Starvation has been shown to effect the rumen microbiome structure [22], leading to our hypothesis that changes in the rumen microbiome following starvation are linked to a reduced rumen fibrolytic capacity. Strategies that combine feeding with the administration of fibrolytic "key species" during realimentation may improve hydrolysis and fermentation of plant polysaccharides and overall health and well-being of the host animal. The rumen contains multiple metabolic niches, and despite the diversity of rumen microbes a limited number of bacterial species is believed to carry out the key function of cellulose deconstruction [32]. Probiotic bacteria are used in the bovine rumen to support productivity in the host organism [16] and combat diarrheal syndromes [1]. In this current pilot study we have investigated whether dosing of cellulolytic bacteria might be used to positively affect the microbial community and the overall microbial fibrolytic activity within the reindeer rumen microbiome during realimentation. We have focused on a proficient cellulose-degrading isolate originating from the reindeer rumen as a candidate probiotic, namely Ruminococcus flavefaciens isolate 8/94-32 (Family Ruminococcaceae) [30]. Ruminal dosing of fibrolytic Ruminococcus to enhance fibre digestion has previously been tested in domestic sheep ( $R$ albus and $R$. flavefaciens; [17]) and cattle (R. flavefaciens; [7]) with varying success. To gain further insight into the impact of bacterial dosing on rumen biomass converting capacity and microbiome structure, and to evaluate the 
potential to improve feeding regimes for reindeer in particular, we have conducted a starvation and realimentation trial with reindeer. The effect of probiotic manipulations on the structure dynamics of the reindeer rumen microbiome were characterized using 454-pyrosequencing of bacterial $16 \mathrm{~S}$ rRNA gene amplicons (V1-V3 region), and changes in the fibrolytic capacity of the rumen simultaneously monitored using functional assays.

\section{Materials and methods}

Animals and diets

Three castrated male reindeer (aged 2 years, initial body mass $63.5-73.5 \mathrm{~kg}$ ) with rumen cannula (U1039 Cannula Sheep 62mm, Macam Rubber, NSW, Australia) were maintained at the animal housing facilities at the University of Tromsø, Norway. The animals had been accustomed to handling, and before the study started they were placed in indoor boxes, where they were kept at temperature $8^{\circ} \mathrm{C}-17^{\circ} \mathrm{C}$ at simulated natural light-darkness cycles $\left(70^{\circ} \mathrm{N}\right)$ during the trials. Animals were treated against parasites with fenbendazol (Panacur vet., Intervet International B.V., Boxmeer, Netherland), and the level of parasites was monitored by faecal sample analyses to avoid untreated parasite infections during trials. The animals were offered commercially produced reindeer feed pellets (grass meal $35.0 \%$, ground oats $26.0 \%$, ground barley $17.5 \%$, sea weed meal $13.0 \%$, molasses $5.0 \%$, vegetable fat $2.0 \%$, silage protein concentrate $1.5 \%$; FK Reinfôr, Felleskjøpet Trondheim, Norway) all days, except days of food deprivation (see "Animal experimental procedures"). Fresh water was available ad libitum at all times.

\section{Animal experimental procedures}

All animals received identical treatment throughout the experiment, and samples from the three animals were processed at the same time. Before the experiments started, measurements were carried out to monitor the background levels of rumen $\mathrm{pH}$, fibre digestion in the rumen (dry matter disappearance (DMD); see below) and rumen bacterial composition (Background, before 4 days of food deprivation and dosing). The experiment was divided into two parts, hereby referred to as Part A (Control / C) and Part B (Treatment / T), which were conducted consecutively in time only separated 
by a four days break during which the animals received water and food ad libitum. Part A of the experiment included dosing the animals with non-inoculated bacterial medium (placebo), while Part B included dosing the animals with bacterial suspensions (prepared and administered as described in the following section). Rumen sampling for 454-pyrosequencing and fibre digestion measurements was carried out at 09:00 and those for $\mathrm{pH}$-measurements were at 09:00 and 14:00 on each of the time points indicated in Fig. 1, as described in the following section. Ruminal dosing was carried out immediately after sampling (Fig.1). The animal experiment was carried out in accordance with regulations in the Norwegian Animal Welfare Act, and approved by the National Animal Research Authority of Norway (FOTS ID 375).

\section{Bacterial cultures and dosing}

A viable inoculum of $R$. flavefaciens 8/94-32 was prepared growing the bacteria in pure culture on liquid M8P medium [29] in an anaerobic chamber (Coy Laboratory Products, Ann Arbor, MI, USA) with an atmosphere of $\mathrm{N}_{2}(90 \%), \mathrm{CO}_{2}(5 \%)$, and $\mathrm{H}_{2}(5 \%)$. The M8P culture medium contained pelleted reindeer feed $(1 \mathrm{~g} / 100 \mathrm{ml})$ commercially available from FK Reinfôr Felleskjøpet, Norway as substrate for the microbial growth. This was the same feed provided for the experimental animals allowing the bacteria to adapt to the substrate prior to dosing. The pelleted feed provided in the culture medium was sterilized by ionizing radiation from a radioactive cobalt source $\left({ }^{60} \mathrm{Co}\right.$, dose up to 15.000 $\mathrm{Gy} / \mathrm{h}$ ) at the Institute for Energy Technology (Kjeller, Norway) to avoid contamination. After $72 \mathrm{hrs}$ of growth the bacterial culture was examined by microscope to check for purity and density, centrifuged ( $2500 \mathrm{rpm}$ for $10 \mathrm{~min})$, and approximately two-thirds of the supernatant was removed to obtain as high a concentration of the viable inoculum of $R$. flavefaciens as possible. Each animal was dosed with $250 \mathrm{ml}$ of the concentrated bacterial suspension through the rumen cannula immediately after rumen sampling on the days indicated in Fig. 1.

\section{Rumen sampling and $\mathrm{pH}$ measurements}

Samples of rumen content were collected through the rumen cannula by suction using a rigid plastic tube (diameter $15 \mathrm{~mm}$ ) attached to a manual suction device. The plastic tube was inserted several times 
at different angles and depths, and the collected rumen content transferred to a beaker in which the rumen $\mathrm{pH}$ was measured using a portable pH-meter (PHM201 with PHC2005 electrode, MeterLab, Radiometer Analytical, France) immediately after sampling. Rumen samples were kept on ice until they were transferred into an anaerobic chamber (within 40min after sampling) where subsamples (approx. 20g) were transferred to vials stored at $-80^{\circ} \mathrm{C}$ until DNA extraction.

\section{Rumen fibrolytic activity}

Ruminal capacity to digest fibre was determined by analysing dry matter disappearance (DMD) of the commercially produced feed pellets and grinded cellulose filter paper (Whatman no.1) based on the in situ nylon bag technique described by Ørskov et al [38]. The filter paper was ground to pass through a 2mm sieve (Cyclotec 1093 Sample mill, Foss tecator, Denmark). Substrates (approx. 2g pellets or 1g filter paper) were placed in nitrogen-free polyester bags (ANKOM Rumen sampling bags, pore size $50(+/-15) \mu \mathrm{m}, 5 \times 10 \mathrm{~cm}$; BarDiamond, ID, USA) and dried overnight at $45^{\circ} \mathrm{C}$, before bags were weighed, labelled and sealed (Super-Seal Bag Sealing Machine, Jencons Scientific, PA, USA). DMDmeasurements were carried out in triplicates, with three bags containing grinded Whatman filter paper (cellulose DMD) and three bags containing pellets (pellet DMD) incubated in the rumen of each animal at each time point. The bags were attached to a thin nylon tube at intervals of approx. $8 \mathrm{~cm}$ distance (one string of each substrate), and incubated for 24 hours. After incubation, the bags were washed in a washing machine (without detergent) at $40^{\circ} \mathrm{C}$ ("wool program", without centrifugation) and dried at $45^{\circ} \mathrm{C}$ for 48 hours, before re-weighing. Negative controls were generated by placing bags in $\mathrm{dH}_{2} \mathrm{O}$ for 24 hours, after which the bags were treated identically to the incubated bags. The DMD is the difference in dry weight before and after ruminal incubation, corrected for the change in DM observed in the negative controls. At days 3, 5, 12 and 16, one to three in situ bags were ripped and hence excluded from the calculations (see Footnote in Fig.2).

\section{Cell harvesting and DNA extraction}

Cells from the liquid phase and particle fraction of whole rumen content samples were harvested from sample aliquots (approx. 0.75-0.85g) in a protocol that included washing with DDS buffer (35) and 
spinning, based on a procedure outlined in Kang et al [15]. In brief, whole rumen contents were resuspended in DDS buffer and subjected to low speed centrifugation. From the supernatant, the dissociated cells from the particle fraction along with cells in the liquid fraction were harvested and concentrated by high-speed centrifugation. The dissociation and harvesting steps were repeated four times for each sample to increase the amount of cells harvested. Harvested cells were then washed with $10 \mathrm{mM}$ Tris- $\mathrm{HCl}, 1 \mathrm{M} \mathrm{NaCl}$, before extraction of DNA as described by Rosewarne et al [25] with only minor alterations. Lysis of cells was carried out by incubation in lysis buffer (RBB+C lysis buffer [37] at $70^{\circ} \mathrm{C}$ for $20 \mathrm{~min}$ with gentle mixing and no beating, followed by precipitation with $\mathrm{CTAB}$ (cetyltrimethyl ammonium bromide) buffer. The mixture was then treated with chloroform, followed by treatment with phenol / chloroform / isoamyl alcohol, before DNA was recovered using isopropanol precipitation. DNA was stored at $-20^{\circ} \mathrm{C}$ until further analysis.

\section{4-pyrosequencing}

The variable region V1-V3 of the 16S rRNA gene of bacteria was amplified using the forward primer (5'- CCT ATC CCC TGT GTG CCT TGG CAG TCT CAG CAA CAG CTA GAG TTT GAT CCT GG -3'), which contains the 454 Life Sciences primer B sequence and the broadly conserved bacterial primer 27F, and the reverse primer (5'-CCA TCT CAT CCC TGC GTG TCT CCG ACT CAG NNN NNN NNT TAC CGC GGC TGC T -3'), which contains the 454 Life Sciences primer A sequence, the broadly-conserved bacterial primer 515R and a unique 8-nt multiplex identifier used to tag each amplicon [13] (Table S1). PCR amplifications were carried out in 50 $\mu 1$ reaction volumes containing $25 \mu 1$ iProof High-Fidelity DNA Polymerase (Bio-Rad, Hercules, CA, USA), $1 \mu 1$ of each primer, $1 \mu 1$ of DNA, and $22 \mu 1$ sterile water. The PCR conditions were as follows: initial denaturation at $98^{\circ} \mathrm{C}$ for $1 \mathrm{~min}$, followed by 25 cycles of $30 \mathrm{sec}$ at $98^{\circ} \mathrm{C}$ (denaturing), $45 \mathrm{sec}$ at $55^{\circ} \mathrm{C}$ (annealing), $45 \mathrm{sec}$ at $72^{\circ} \mathrm{C}$ (extension), and a final extension of $7 \mathrm{~min}$ at $72^{\circ} \mathrm{C}$. Amplicons were checked for size and purity by electrophoresis on a $1 \%$ agarose gel, and concentrations were measured using Quant-iT dsDNA BR Assay Kit (Invitrogen, Carlsbad, CA, USA). All PCR products were mixed in equal molar amounts, and the pooled sample was run on a $1 \%$ agarose gel, from which the pooled PCR product band was excised and purified using the NucleoSpin Extract II kit (Macherey-Nagel, Düren, Germany). The 
454/Roche GS FLX sequencing was carried out using the LIB-A chemistry at the Norwegian HighThroughput Sequencing Centre (Oslo, Norway). Sequences have been deposited in the NCBI Sequence Read Archive (SRA) under the accession number SRA051253 and BioProject 89735.

16S rRNA gene sequence analysis

The 16S rRNA gene sequences were processed using the QIIME software package [5] and removed from the analysis if they were $<350$ or $>550$ nt in length, contained ambiguous bases, had a mean quality score $<25$, contained a homopolymer run exceeding $6 \mathrm{nt}$, or did not contain a primer or barcode sequence. Error-correction, chimera removal and operational taxonomic unit (OTUs) clustering was performed using USEARCH quality filtering with QIIME, which incorporates UCHIME [10] and a 97\% sequence identity threshold. Prior to comparison of $16 \mathrm{~S}$ rRNA gene sequences within different sampling points, each dataset was randomly "subsampled" using QIIME to normalize each dataset and remove sample heterogeneity (1075 sequences were used for each animal at each time-point). Evenness was measured using the Simpson index in QIIME. The most abundant sequence in each OTU was chosen as the representative sequence. Representative sequences were aligned against the Greengenes core set [9] using PyNAST software [4] with a minimum alignment length of 150 and a minimum identity of $75 \%$. Taxonomy was assigned to each OTU using the Ribosomal Database Project (RDP) classifier [8] with a minimum support threshold of $80 \%$ and the RDP taxonomic nomenclature. The alignment was filtered to remove gaps and hypervariable regions using a Lane mask, and a maximum-likelihood tree was constructed from the filtered alignment using FastTree [24]. Phylogenetic trees were used to measure Phylogenetic Distance (PD), and construct unweighted UniFrac distance matrices [19] which were visualised using principal coordinates analysis. Network maps were used to visualize correlations (Pearson's correlation) between genera with more than $0.5 \%$ relative abundance and environmental variables $(\mathrm{pH}$, cellulose and pellet DMD). This previous filtering step removed poorly represented genera and reduced network complexity. Linear regressions were used to test the relationships between alpha diversity metrics and $\mathrm{pH}$ with pellet DMD. 


\section{Statistics}

DMD measures, $\mathrm{pH}$ measures and calculations of abundance, Phylogenetic Distance and evenness are presented as means \pm standard deviation (SD) $(n=3)$. DMD data were analysed using ANOVA mixed model in XLSTAT (Addinsoft SARL), with two fixed effects (treatment and day) with interaction, and one random effect (animal). Statistical significance was declared at $p<0.05$.

\section{Results and Discussion}

Dosing inflicts changes in rumen microbiome function and $\mathrm{pH}$

Ruminal dosing of a highly fibrolytic $R$. flavefaciens did not increase the digestibility during realimentation in reindeer. On the contrary, compared to dosing of non-inoculated medium, dosing of the bacterial suspension led to a decrease in the cellulose DMD $(-11.6 \%, p=0.03,95 \%$ CI $[-22.0,-1.2])$ and pellet DMD $(-3.7 \%, p=0.03,95 \%$ CI $[-7.0,-0.3])$ when measured eight days after the last ruminal dosing (Day 19 of each part of the trial) (Fig. 2a and b). In a study with repeated ruminal dosing of Ruminococcus species to adult sheep fed low quality Rhodes grass, Krause et al [17] reports of no improvement in digestibility using the in situ nylon bag technique. Another study with repeated dosing of $R$. flavefaciens to cows by Chiquette et al [7] reports that the effect of dosing on digestibility is dependent on diet, with no change in digestibility observed for animals on a high forage diet, and an increase in digestibility of animals on a high concentrate diet (to a level similar to that of high forage fed cows). Thus, although previous studies report absent, or limited improvement in digestibility following dosing, this is, to our knowledge, the first study to report reduction in digestibility after ruminal dosing of a fibrolytic Ruminococcus strain.

To determine whether animal manipulations in the form of feed-deprivation and ruminal dosing affected rumen $\mathrm{pH}$ levels, measurements were taken at all sample-points throughout the study (Fig. 1). Reindeer rumen $\mathrm{pH}$ measurements were elevated during starvation (Fig. 2c) to levels previously observed in feed deprived animals [28, 39]. Dosing and realimentation resulted in a return to background levels that was similar in both control and treatment periods, however several minor differences were noted. During control-dosing a reduction of rumen $\mathrm{pH}$ was observed and a gradual return to background levels was consistent with findings of Sletten and Hove [28]. Probiotic-dosing 
resulted in an additional moderate drop in $\mathrm{pH}$ of approx. 0.6 from control levels, and the lowest recording was five days after previous findings which measured lowest $\mathrm{pH}$ levels $1-2$ days after realimentation commences (Fig. 2c). Ruminal pH differed between the two food-deprivation periods (Dep-C/T) being $\sim 0.4$ units lower during this period in the control trial (Fig. 2c). The observed differences in $\mathrm{pH}$ suggest that the rumen microbial community may have been different at the beginning of the placebo dosing and the bacterial dosing. Hence, changes in the microbial communities may consequently not only have been caused by the dosing itself but partly also by temporal fluctuations in the microbial community as reflected in the $\mathrm{pH}$ (Fig. 2c).

Rumen microbiome structure influenced by dosing Community analysis was carried out based on a total of 108250 non-chimeric 454-generated reads. The decrease of species evenness in Dos-T samples was presumably caused by a pronounced increase in the genus Prevotella that approximately doubled in relative abundance during the period $(>50 \%$ of the total community: Fig. 2d). Comparison of alpha and beta diversity metrics illustrated that rumen manipulation in the form of bacterial dosing influenced rumen microbiome structure. Measurements of Phylogenetic Distance (PD) (the sum of all branch lengths in a 16S rRNA gene phylogenetic tree: [12]) indicated that highest sample diversity was calculated for the background sample, whilst PD decreased to its lowest levels during probiotic treatment and to a lesser extent during control dosing periods (Fig. 2e). Post-dosing PD measurements showed that the diversity returned to levels similar to those observed prior to dosing, in both the control and the treatment experiment. Species evenness (the relative abundance of different species in the community) measured via the Simpson's index was also observed to be lowest during dosing with bacteria (Fig. 2f), after which it recovered to pre-dosing levels.

The factors driving phylogenetic variation in microbial communities between different samples was also determined via principle coordinate analysis (PCoA) of unweighted UniFrac matrices [18] (Fig. 3). Analysis of the total microbiome community showed that samples taken during probiotic dosing (Dos-T) exhibited the largest distance from background samples and thus greatest change in phylogenetic structure (Fig. 3a). Interestingly, control dosing (Dos-C) caused similar phylogenetic 
variance in two of the three animals, suggesting the process of dosing administration regardless of the

presence of the probiotic is the predominant source of variation in the data. Fig. 3a also inferred clustering by individual animal (e.g. Animal_09), highlighting that distinct lineages are present in each animal and are a source of variation. Inter-animal variation in microbiome structure, has previously been observed in other young [18] and adult ruminants [3, 33], even when fed the same diet and receiving the same treatment. Unweighted Unifrac matrices were additionally calculated with animals collectively considered at each time-point to accommodate for inter-animal variation and make the effects of control vs. treatment more evident (Fig. 3b). Analysis based on pooled microbiota confirms that distinct microbiome disturbances are caused by the act of dosing administration, in addition to revealing a clear response of the microbiome in the subsequent periods after dosing (pDos1-2). The microbiome community in control samples changed directionally until it returned to a structure moresimilar to the background and feed-deprived states. Similar directional patterns were also observed in treatment samples albeit with an overall greater distance from background samples, further highlighting the greater variation caused by probiotic dosing. Interestingly both pDos-C-2 and pDos$\mathrm{T}-2$ clustered in close proximity, suggesting the recovery of microbiome community structure in response to dosing with either a placebo or probiotic was similar.

Comparison of OTUs against the Ribosomal Database Project demonstrated that the reindeer rumen microbiota was dominated by the phyla Bacteroidetes (54.5-77.6\%) and Firmicutes (18.5-35.7\%) (Fig. 4), which exhibits compositional consistency with previous metagenomic analysis described from the Svalbard reindeer rumen microbiome [23]. Family level analysis showed that Prevotellaceae (24.2$67.5 \%$ ), and Ruminococcaceae (3.7-19.4\%) dominated each sample, as did uncharacterised groups affiliated to the phylum Bacteroidetes and the orders Bacteroidales and Clostridiales (Fig. 4). There was a strong influence of ruminal dosing on the composition of different microbial lineages (Dos- $\mathrm{T}$ in Fig. 4). The most evident changes in relative abundance were an increase in Prevotellaceae (predominantly the genus Prevotella: Fig. 2d) and decrease of uncharacterised Bacteroidetes phylotypes (uBacNR). Interestingly the relative abundance of the Ruminococcaeceae, the affiliate family of the dosed $R$. flavefaciens strain, remained at a similar level throughout the experiment (Fig. 2d). However all $R$. flavefaciens strain 8/94-32 -affiliated OTUs were below detection levels post- 
dosing (Table S2), suggesting that the introduced bacterium did not persist in the rumen 72 or 96 hours after dosing. Absolute numbers for OTUs affiliated to $R$. flavefaciens, $R$. albus and $F$. succinogenes indicate dosing did not stimulate other closely related cellulolytic strains (Table S2).

Relating microbiome structure to function and $\mathrm{pH}$

To gain insight into how community structure relates to function, we investigated for relationships between bacterial diversity and composition towards cellulose and pellet DMD. No clear correlation between diversity (PD) or evenness and cellulose DMD was observed (R: 0.30, p: 0.1 and R: 0.31, p: 0.1 respectively). However communities with higher diversity and evenness functioned more efficiently with respect to pellet DMD (Fig. 5a-b). Observations linking higher phylogenetic variation and evenness to improved community efficiency have been previously recognized in anaerobic biogas reactors $[34,36]$. Both metrics are believed to play an important role in preserving the functional stability of microbial ecosystems via a higher capacity to use redundant functional pathways that enable more efficient responses to environmental changes [34]. A similar effect may be applicable to the reindeer rumen microbiome, an anaerobic ecosystem that exhibits similar metabolic properties to a biogas reactor (i.e. fibre hydrolysis and gas production) and which experiences environmental changes that affects microbial composition and functions.

Correlation analysis deduced that no relationships were observed with dominant Bacteroidetes genera and cellulose DMD, however a positive correlation was observed between uncharacterized members of the Veillonellaceae (Fig. 5d), a Firmicutes-affiliated family that has not been previously associated with known cellulolytic bacteria. The genera Ruminococcus and Fibrobacter had no observed relative abundance relationships with cellulose DMD. This result was not entirely unexpected given that the relative abundance of all OTUs exhibiting similarity ( $>97 \%)$ to known cellulolytic species Fibrobacter succinogenes, $R$. albus and $R$ flavefaciens were all on the lower limit of detection and collectively amounted to less than $0.4 \%$ (Table S2).

Based on pellet components it is expected that high levels of protein, starch, soluble sugars and plant polysaccharides including cellulose and hemicellulosic substrates such as beta-glucans, arabinoxylans were readily available to the resident microbiome. The relative abundance of Bacteroidetes-affiliated 
lineages displayed both negative (Prevotella) and positive ( $\mathrm{uBacNR}$ ) relationships with pellet DMD (Fig. 5d). The Bacteroidetes are specialized in the breakdown of complex plant polysaccharides and uncharacterised lineages have previously been described in the rumen of Svalbard reindeer that harbour enzymatic capabilities towards plant polysaccharides such as beta-glucans and arabinoxylans [20]. Negative associations between Prevotella and pellet DMD align with earlier ruminant studies that detect high levels of Prevotella simultaneous with negative correlations towards DMD [6]. Both findings are somewhat surprising since rumen Prevotella spp. have a renowned metabolic versatility, capable of utilizing a wide variety of proteins, peptides and monosaccharides as well as plant polysaccharides including starch, hemicellulose, cellodextrins and pectin [2]. Firmicutes-affiliated genera Butyrivibrio and Pseudobutyrivibrio both demonstrated positive relationships with pellet DMD. Representatives for both Butyrivibrio and Pseudobutyrivibrio have been isolated from the rumen of Norwegian reindeer and demonstrate activity against xylan and carboxymethyl-cellulose [30].

Surprisingly no significant relationships were observed between Prevotella and $\mathrm{pH}$ (R: 0.356, p: 0.067) which was at its highest relative abundance levels when $\mathrm{pH}$ was lowest (Fig. 2c), although the relative abundance of its affiliate family Prevotellaceae did correlate positively to $\mathrm{pH}(\mathrm{R}: 0.528$, $\mathrm{p}<0.01)$. An important phenotype of ruminant Prevotella spp. is their remarkable capacity to grow efficiently at relatively low $\mathrm{pH}$ values [27]. Overall, negative correlations were observed between $\mathrm{pH}$ and pellet DMD (Fig. 5c) which is in agreement with in vitro and in vivo studies that indicate that fibre digestion can be severely inhibited by even modest declines in ruminal $\mathrm{pH}$ [26]. Cellulolytic rumen bacteria including $R$. flavefaciens, $F$. succinogenes and $R$. albus as well as the efficient saccharolytic bacterium Butyrivibrio fribrisolvens are highly sensitive to drops in $\mathrm{pH}$ [26]. In this study, all genera that correlated positively to pellet DMD had negative relationships to $\mathrm{pH}$ including the dominant uBacNR group and Butyrivibrio (Fig. 5d). Although only moderate, more acidic ruminal conditions were associated with dosing and could explain the lower digestibility of cellulose and dry matter in the pellet feed. Moreover the interconnections between dosing, $\mathrm{pH}$ and high Prevotella remain to be elucidated i.e. does treatment dosing cause a drop in $\mathrm{pH}$ thus providing a niche for 
Prevotella to dominate, or does dosing result in a community shift towards Prevotella dominance whose fermentative metabolism cause $\mathrm{pH}$ levels to decline?

Probiotic dosing does not stimulate $R$. flavefaciens levels or DMD in the reindeer rumen A probiotic effect of dosed fibrolytic bacteria through increased fibre digestion is dependent on the dosed bacteria being able to establish in the rumen, even if only for a short time during realimentation. Previous attempts of Ruminococcus spp. dosing to adult ruminants has repeatedly demonstrated that the introduced bacteria rapidly declines post-dosing or does not persist. In the current study the animals were food deprived prior to dosing administration, which is an important difference in conditions from previous dosing studies. Viable cell counts of rumen contents from reindeer have previously shown that starvation for four days reduces the total viable populations of anaerobic bacteria in the rumen fluid by as much as $99.7 \%$ and bacteria adhering to the rumen solids by $95.9 \%$ [39]. A severe reduction in rumen microbes could result in "open ecological niches" to colonize, analogous to the immature rumen where rumen probiotic studies have experienced success $[7,16]$. However, community analysis shows that the starvation period did not result in an unstable microbial community with only minimal changes in phylogenetic structure observed (Fig. 3). Functional studies confirmed a limited DMD effect during starvation periods (Fig. 2) and rumen function did not seem severely depressed. The latter is supported by the observation that the animals did not show any signs of diarrhoea during realimentation. Since the reindeer in this study were managing surprisingly well with the food deprivation, it is possible the opportunities for introduced bacteria to establish in the rumen were limited.

In conclusion, probiotic bacteria and their influences in combating digestive disorders in humans and animals have been previously demonstrated, albeit with inconsistent measures of success in ruminants. Although this current probiotic effort to enhance feed fermentation in reindeer during ralimentation did not succeed to expectations, the community-wide analysis enabled an opportunity to measure microbiome structural responses to rumen manipulation via dosing. 16S rRNA gene analysis illustrates that dosing of a fibrolytic bacterium drives phylogenetic variance within reindeer rumen 
microbiome structure, which ultimately results in reduced rumen biomass saccharolytic capacity. Interestingly the introduction of a gram-positive Firmicutes-affiliated bacterium largely impacted on dominant gram-negative Bacteroidetes-affiliated bacteria including uBacNR and Prevotella. Uncharacterised $\mathrm{uBacNR}$ was positively correlated to pellet DMD, which reinforces previous metagenomic analysis that infer the major involvement of deeply branched Bacteroidetes in fibre hydrolysis in the reindeer rumen. Given the dominance of Prevotella in instances of lower pellet DMD we speculate Prevotella metabolism to be directed towards utilizing soluble sugars and proteins in instances of rumen disturbance (i.e. dosing). Finally, UniFrac data suggests that ruminal manipulations via cannula entry cause disruptions in the microbiome structure irrespective of placebo or treatment dosing. Our results, therefore, suggest that future rumen studies that incorporate cannula-based experiments need to consider, during the design and analysis stages, the potential for this process to impact the structure and function of the rumen microbiome.

\begin{abstract}
Acknowledgments The authors thank Prof. Arnoldus Schytte Blix for help with the rumen fistulation of the animals, Hans Edvin Lian for all help with animals, Alexandra Heuer for help with sampling during animal experiments, and Prof. Michael Greenacre for help with DMD statistics. This study is linked to the framework of the International Polar Year (IPY) as part of the consortium IPY \# 399 EALAT: Climate change and reindeer husbandry. Funding was provided by the Reindeer Husbandry Research Fund, University of Tromsø, Roald Amundsen Centre for Arctic Research (University of Tromsø) and Centre for Sami Studies (University of Tromsø). PBP is supported by The Research Council of Norway's FRIPRO program (214042) and the European Commission Marie Curie International Incoming Fellowship (PIIF-GA-2010-274303).
\end{abstract}

\title{
References
}

1. Abe F, Ishibashi N, Shimamura S (1995) Effect of administration of bifidobacteria and lactic acid bacteria to newborn calves and piglets. J Dairy Sci 78:2838-2846

2. Avgustin G, Wallace RJ, Flint HJ (1997) Phenotypic diversity among ruminal isolates of Prevotella ruminicola: proposal of Prevotella brevis sp. nov., Prevotella bryantii sp. nov., and 
Prevotella albensis sp. nov. and redefinition of Prevotella ruminicola. Int J Syst Bacteriol $47: 284-288$

3. Belenguer A, Toral PG, Frutos P, Hervás G (2010) Changes in the rumen bacterial community in response to sunflower oil and fish oil supplements in the diet of dairy sheep. J Dairy Sci 93:32753286

4. Caporaso JG, Bittinger K, Bushman FD, DeSantis TZ, Andersen GL, Knight R (2010) PyNAST: a flexible tool for aligning sequences to a template alignment. Bioinformatics 26:266-267

5. Caporaso JG, Kuczynski J, Stombaugh J, Bittinger K, Bushman FD, Costello EK, Fierer N (2010) QIIME allows integration and analysis of high-throughput community sequencing data. Nat Meth $7: 335-336$

6. Carberry CA, Kenny DA, Han S, McCabe MS, Waters SM (2012) Effect of phenotypic residual feed intake and dietary forage content on the rumen microbial community of beef cattle. Appl Environ Microbiol 78:4949-4958

7. Chiquette J, Talbot G, Markwell F, Nili N, Forster RJ (2007) Repeated ruminal dosing of Ruminococcus flavefaciens $\mathrm{NJ}$ along with a probiotic mixture in forage or concentrate-fed diary cows: Effect of ruminal fermentation, cellulolytic populations and in sacco digestibility. Can J Anim Sci 87:237-249

8. Cole JR, Chai B, Marsh TL, Farris RJ, Wang Q, Kulam SA, Chandra S, McGarrell DM, Schmidt TM, Garrity GM, Tiedje JM (2003) The Ribosomal Database Project (RDP-II): previewing a new autoaligner that allows regular updates and the new prokaryotic taxonomy. Nucleic Acids Res $31: 442-443$

9. DeSantis TZ, Hugenholtz P, Larsen N, Rojas M, Brodie EL, Keller K, Huber T, Dalevi D, Hu P, Andersen GL (2006) Greengenes, a chimera-checked 16S rRNA gene database and workbench compatible with ARB. Appl Environ Microbiol 72:5069-5072

10. Edgar RC, Haas BJ, Clemente JC, Quince C, Knight R (2011) UCHIME improves sensitivity and speed of chimera detection. Bioinformatics 27:2194-2200 
11. Eira IM, Jaedicke C, Magga OH, Maynard NG, Vikhamar-Schuler D, Mathiesen SD (2013) Traditional Sámi snow terminology and physical snow classification - Two ways of knowing. Cold Regions Science and Technology 85:117-130

12. Faith DP (1992) Conservation evaluation and phylogenetic diversity. Biol Conserv 61:1-10

13. Hamady M, Walker JJ, Harris JK, Gold NJ, Knight R (2008) Error-correcting barcoded primers for pyrosequencing hundreds of samples in multiplex. Nat Meth 5:235-237

14. Josefsen TD, Sørensen KK, Mørk T, Mathiesen SD, Ryeng KA (2007) Fatal inanition in reindeer (Rangifer tarandus tarandus): Pathological findings in completely emaciated carcasses. Acta Vet Scand 49:27

15. Kang S, Denman SE, Morrison M, Yu Z, McSweeney CS (2009) An efficient RNA extraction method for estimating gut microbial diversity by polymerase chain reaction. Curr Microbiol $58: 464-471$

16. Kmet V, Flint HJ, Wallace RJ (1993) Probiotics and manipulation of rumen development and function. Arch Tierernahr 44:1-10

17. Krause DO, Bunch RJ, Colan LL, Kennedy PM, Smith WJ, Mackie RI, McSweeney CS (2001) Repeated ruminal dosing of Ruminococcus spp. does not result in persistence, but changes in other microbial populations occur that can be measured with quantitative 16S-rRNA-based probes. Microbiology 147:1719-1729

18. Li RW, Connor EE, Li C, Baldwin VRL, Sparks ME (2012) Characterization of the rumen microbiota of pre-ruminant calves using metagenomic tools. Environ Microbiol 14:129-139

19. Lozupone C, Knight R (2005) UniFrac: A new phylogenetic method for comparing microbial communities. Appl Environ Microbiol 71:8228-8235

20. Mackenzie AK, Pope PB, Pedersen HL, Gupta R, Morrison M, Willats WG, Eijsink VGH (2012) Two SusD-like proteins encoded within a polysaccharide utilization locus of an uncultured ruminant Bacteroidetes phylotype bind strongly to cellulose. Appl Environ Microbiol 78:59355937 
21. Mathiesen SD, Haga ØE, Kaino T, Tyler NJC (2000) Diet composition, rumen papillation and maintenance of carcas mass in female Norwegian reindeer (Rangifer tarandus tarandus) in winter. J Zool 251: 129-138

22. Nilsson A, Åhman B, Murphy M, Soveri T (2006) Rumen function in reindeer (Rangifer tanandus tarandus) after sub-maintenance feed intake and subsequent feeding. Rangifer 26:73-83

23. Pope PB, Mackenzie AK, Gregor I, Smith W, Sundset MA, McHardy AC, Morrison M, Eijsink VGH (2012) Metagenomics of the Svalbard reindeer rumen microbiome reveals abundance of polysaccharide utilization loci. PLoS ONE 7:e38571.

24. Price MN, Dehal PS, Arkin AP (2010) FastTree 2 - approximately maximum-likelihood trees for large alignments. PLoS One 5:e9490.

25. Rosewarne CP, Pope PB, Denman SE, McSweeney CS, O'Cuiv P, Morrison M (2010) High-yield and phylogenetically robust methods of DNA recovery for analysis of microbial biofilms adherent to plant biomass in the herbivore gut. Microb Ecol 61:448-454

26. Russell JB, Dombrowski DB (1980) Effect of $\mathrm{pH}$ on the efficiency of growth by pure cultures of rumen bacteria in continuous culture. Appl Environ Microbiol 39:604-610

27. Russell JB, Wilson DB (1996) Why are ruminal cellulolytic bacteria unable to digest cellulose at low pH? J Dairy Sci 79:1503-1509

28. Sletten H, Hove K (1990) Digestive studies with a feed developed for realimentation of starving reindeer. Rangifer 10:31-37

29. Sundset MA, Kohn A, Mathiesen SD, Præsteng KE (2008) Eubacterium rangiferina, a novel usnic acid resistant bacterium from the reindeer rumen. Naturwissenschaften 95:741-749

30. Sundset MA, Præsteng KE, Cann IK, Mathiesen SD, Mackie RI (2007) Novel rumen bacterial diversity in two geographically separated sub-species of reindeer. Microb Ecol 54:424-438

31. Tyler NJC, Turi JM, Sundset MA, Bull KS, Sara MN, Reinert E, Oskal N, Nellemann C, McCarthy JJ, Mathiesen SD, Martello ML, Magga OH, Hovelsrud GK, Hanssen-Bauer I, Eira NI, Eira IMG, Corell RW (2007) Saami reindeer pastoralism under climate change: Applying a generalized framework for vulnerability studies to a sub-arctic social-ecological system. Global Env Change 17:191-206 
32. Wallace RJ (2008) Gut microbiology - broad genetic diversity, yet specific metabolic niches. Animal 2:661-668

33. Welkie DG, Stevenson DM, Weimer PJ (2010) ARISA analysis of ruminal bacterial community dynamics in lactating dairy cows during the feeding cycle. Anaerobe 16:94-100

34. Werner JJ, Knights D, Garcia ML, Scalfone NB, Smith S, Yarasheski K, Cummings TA, Beers AR, Knight R, Angenent LT (2011) Bacterial community structures are unique and resilient in full-scale bioenergy systems. Proc Natl Acad Sci USA 108:4158-4163

35. Whitehouse NL, Olson VM, Schwab CG, Chesbro WR, Cunningham KD, Lykos T (1994) Improved techniques for dissociating particle-associated mixed ruminal microorganisms from ruminal digesta solids. J Anim Sci 72:1335-1343

36. Wittebolle L, Marzorati M, Clement L, Balloi A, Daffonchio D, Heylen K, De Vos P, Verstraete W, Boon N (2009) Initial community evenness favours functionality under selective stress. Nature 458:623-626

37. Yu Z, Morrison M (2004) Improved extraction of PCR-quality community DNA from digesta and fecal samples. Biotechniques 36:808-812

38. Ørskov ER, Hovell FDD, Mould F (1980) The use of the nylon bag technique for the evaluation of feedstuffs. Trop Animal Prod 5:195-213

39. Aagnes TH, Sørmo W, Mathiesen SD (1995) Ruminal microbial digestion in free-living, in captive lichen-fed, and in starved reindeer (Rangifer tarandus tarandus) in winter. Appl Environ Microbiol 61:583-591 


\section{Titles and legends to figures}

Figure 1 Animal experimental protocol. All animals underwent identical conditions and sampling procedures at all time. The experiment was divided into two periods, "Part A: Control" (C) and "Part B: Treatment" $(\mathrm{T})$, that included four days of food deprivation, eight days of realimentation and placebo (= medium) or bacterial dosing, respectively, followed by 12 days of feeding post-dosing. Part B was carried out four days after Part A was finished after a four-day break period. Except for dosing content, un-inoculated media in the control period (Dos-C) and a suspension of $R$. flavefaciens strain 8/94-32 in the treatment period (Dos-T), experimental treatments, sampling and measurements were identical in both periods.

Figure 2 Rumen functionality parameters and microbiota community characteristics during the feeding trial. $(a, b)$ Dry matter disappearance (DMD) $(n=3)$ of (a) cellulose (grinded Whatman filter paper) and (b) feed pellets measured using the in situ nylon bag technique. (c) Rumen $\mathrm{pH}(\mathrm{n}=3)$. (d) Relative abundance of Prevotella and Ruminococcaeceae assigned OTUs in the rumen microbiota $(n=3)$. (e) Phylogenetic Distance (PD) $(n=3)$. (f) Evenness (Simpsons index) (n=3). Values are given as means with error bars representing SD. ${ }^{a}$ mean of 8 measurements; ${ }^{b}$ mean of 7 measurements; ${ }^{c}$ mean of 6 measurements.

Figure 3 Phylogenetic distances between samples depicted by principal coordinate analysis (PCoA) of unweighted UniFrac similarities. Samples from reindeer rumen were obtained from animals $(n=3)$ during different feeding regimes and dosing treatment. (a) PCoA including all OTUs. (b) PCoA including OTUs pooled for all animals $(\mathrm{n}=3)$ at the various time points. Back., background; Dep-C, food deprived in control; Dos-C, placebo dosing in control; pDos1-C, first post-dosing in control; pDos2-C, second post-dosing in control; Dep-T, food deprived in treatment; Dos-T, dosing of bacteria; pDos1-T, first post-dosing in treatment; pDos2-T, second post-dosing in treatment.

Figure 4 OTU-based community composition of rumen microbiota of reindeer $(n=3)$ based on 454pyrosequencing sequences assigned to OTUs at a 97\% identity level. Bubble size denotes the relative 
abundance (percentage) of the most abundant bacterial families at each time point. Phyla affiliation is indicated in parenthesis. B: Bacteroidetes, F: Firmicutes, P: Proteobacteria. Back., background; DepC, food deprived in control; Dos-C, placebo dosing in control; pDos1-C, first post-dosing in control; pDos2-C, second post-dosing in control; Dep-T, food deprived in treatment; Dos-T, dosing of bacteria; pDos1-T, first post-dosing in treatment; pDos2-T, second post-dosing in treatment.

Figure 5 The relationships between diversity metrics, metadata and the relative abundances of dominant bacterial genera. (a-c) Linear regressions were used to test the relationships between alpha diversity metrics, $\mathrm{pH}$ and pellet DMD. Panels depict the relationships. PD: Phylogenetic Diversity (d) Correlation network showing the relationships between different bacterial genera and DMD (cellulose and pellet) and $\mathrm{pH}$ within the reindeer rumen. Nodes with a significant Pearson's correlation $(p<0.05)$ are connected by an edge with values indicated: positive correlations are displayed in blue, negative correlations in red. Node sizes of the different phylogenetic groups reflect their relative abundance. uBacNR: unclassified Bacteroidetes group.

\section{Supplementary Information}

Table S1 454-pyrosequencing statistics.

Table S2 Absolute counts for reindeer rumen OTUs affiliated to known cellulolytic strains. 


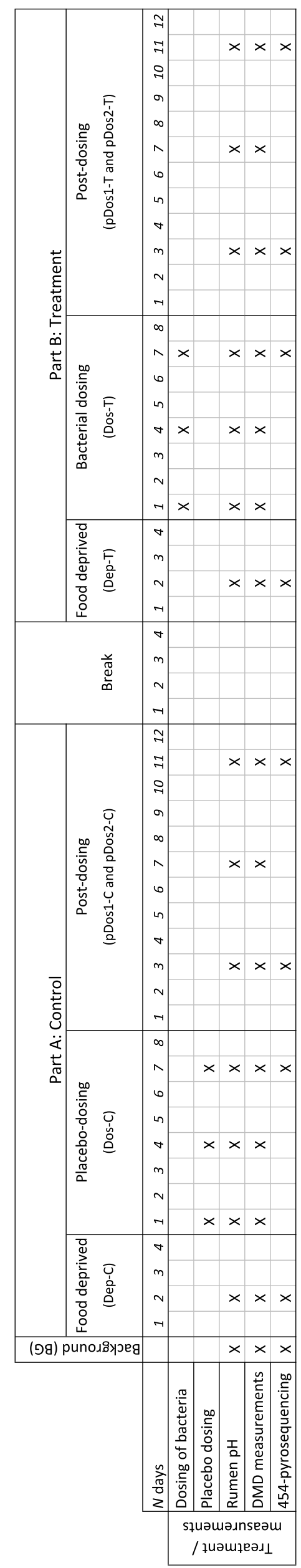



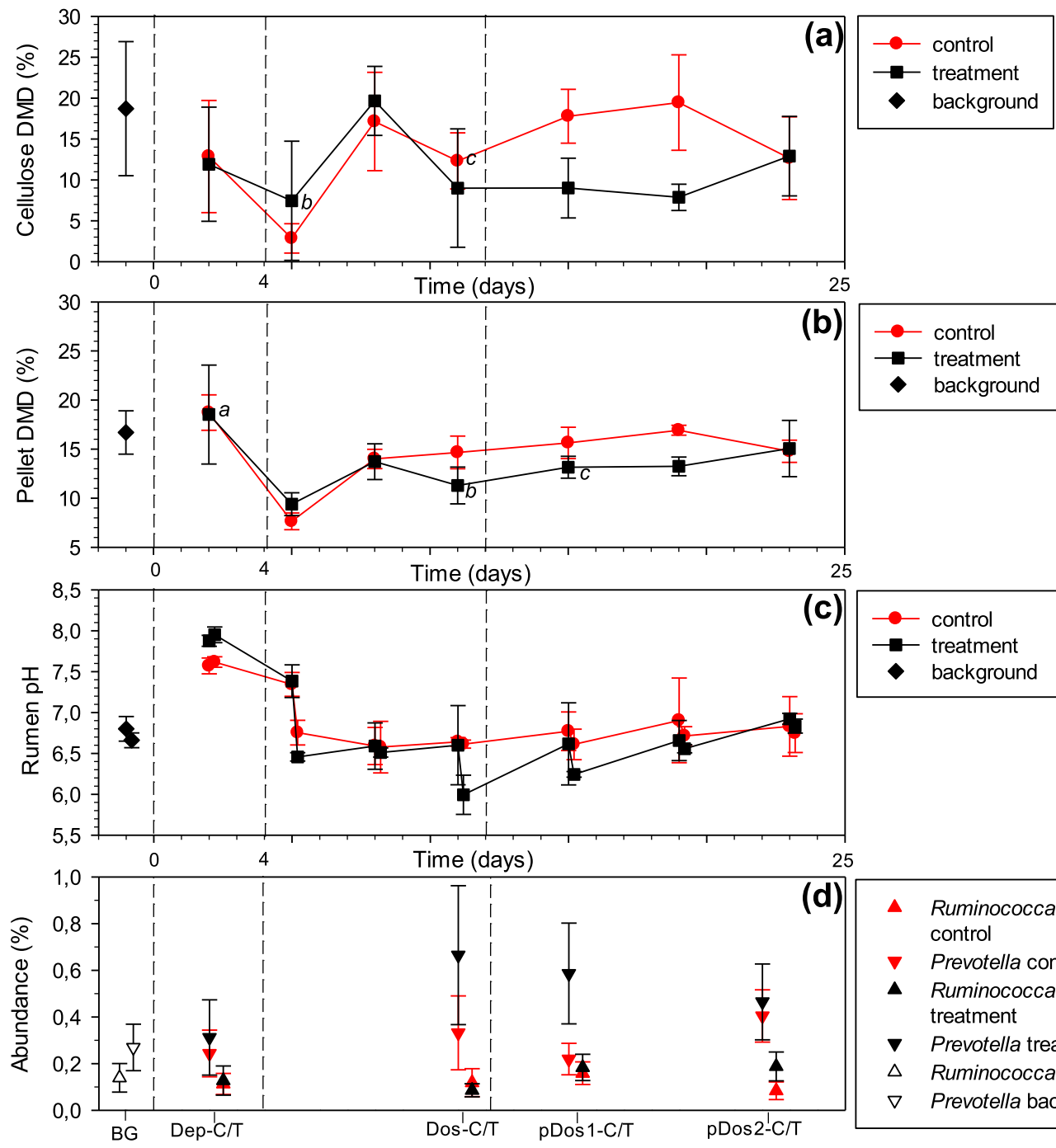

(b)
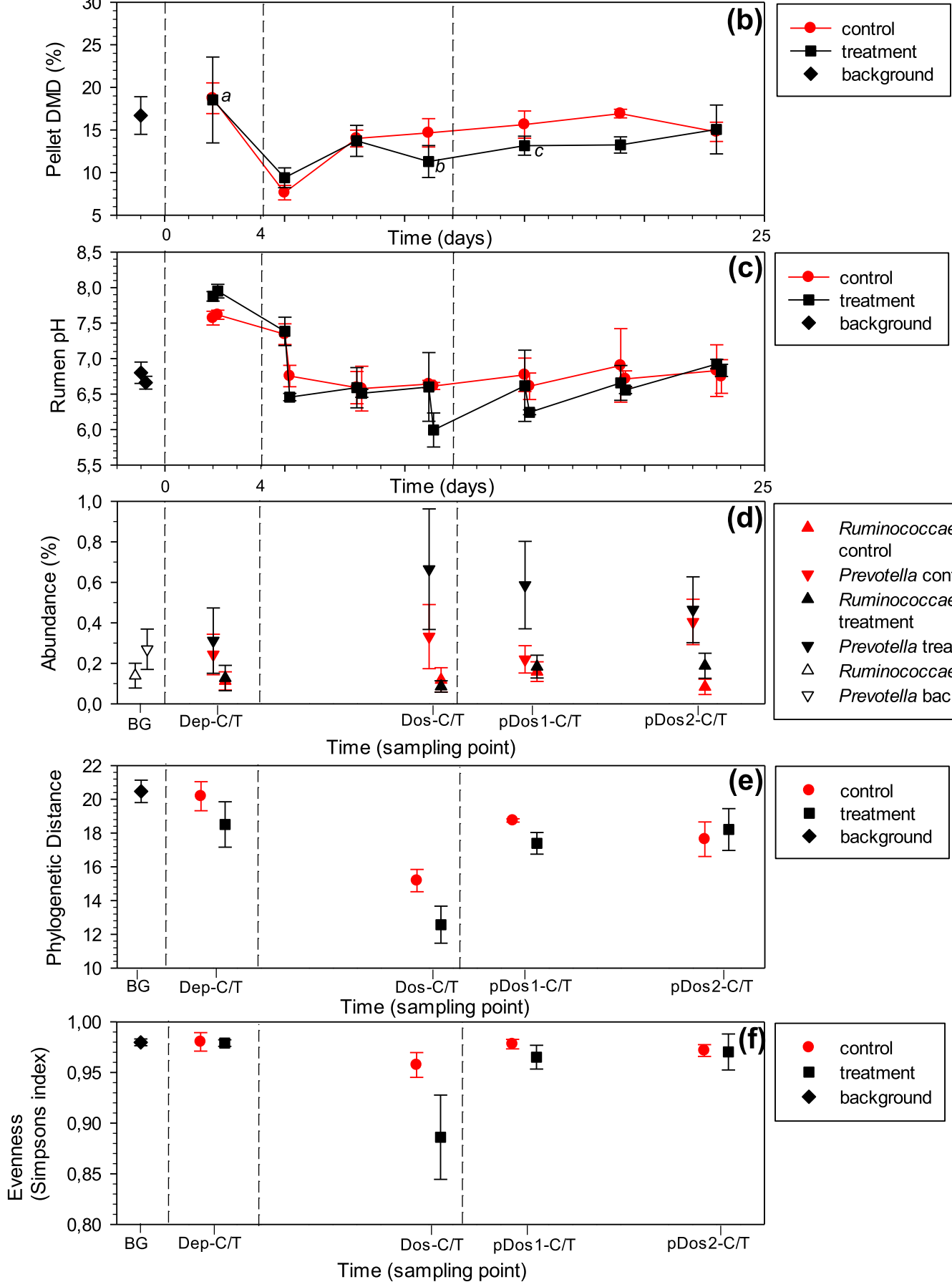

- Ruminococcaeceae control

v Prevotella control

- Ruminococcaeceae treatment

v Prevotella treatment

$\Delta$ Ruminococcaeceae background

$\nabla$ Prevotella background 

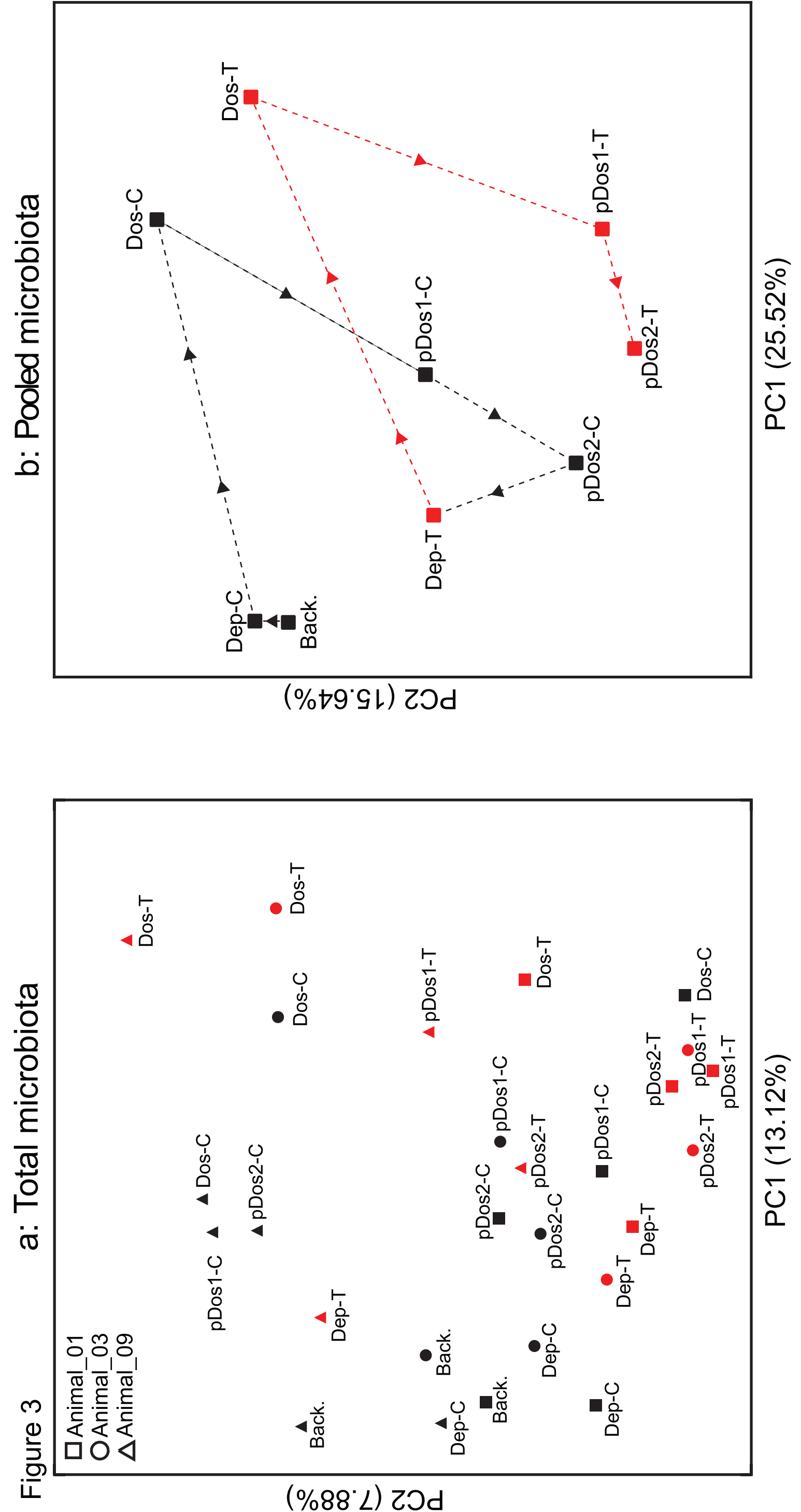

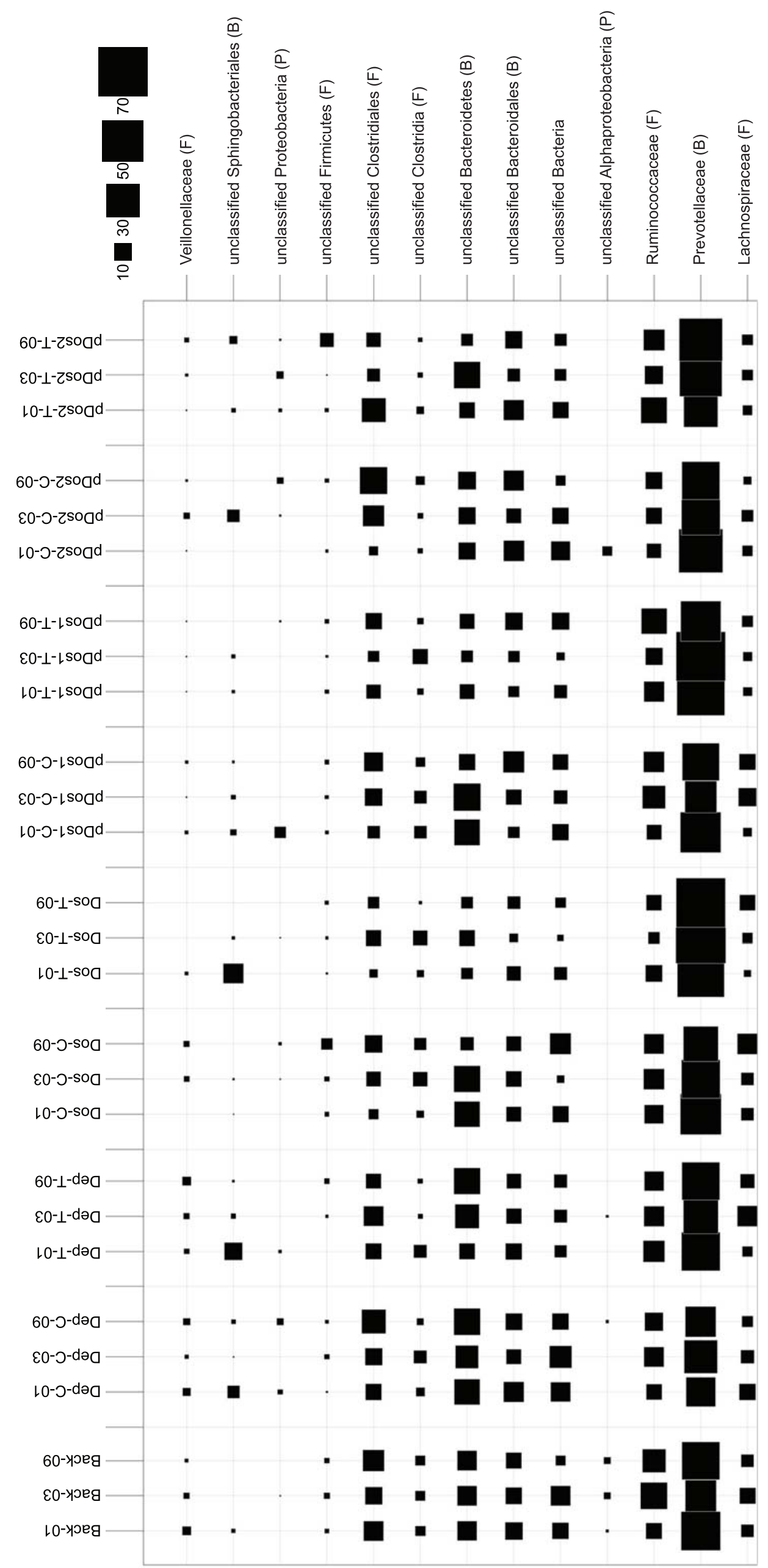

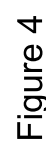


Fiquare 5

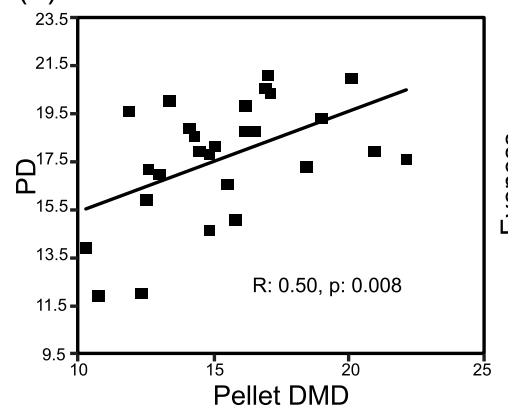

(b)

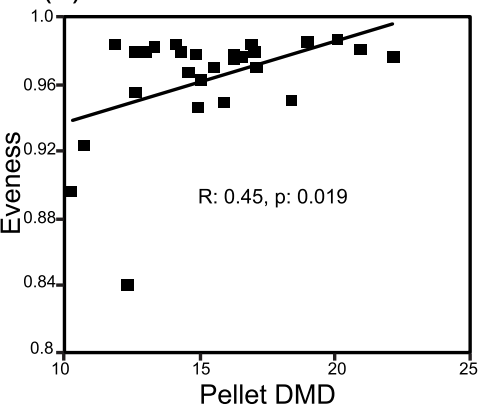

(d)

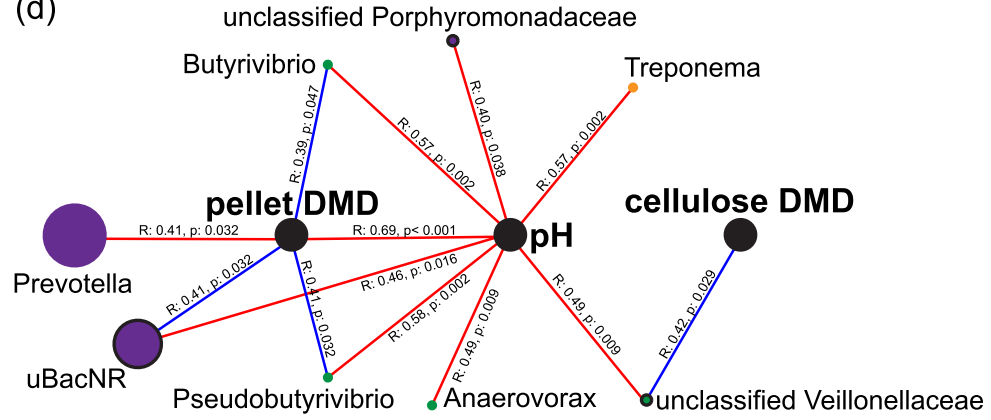

(c)

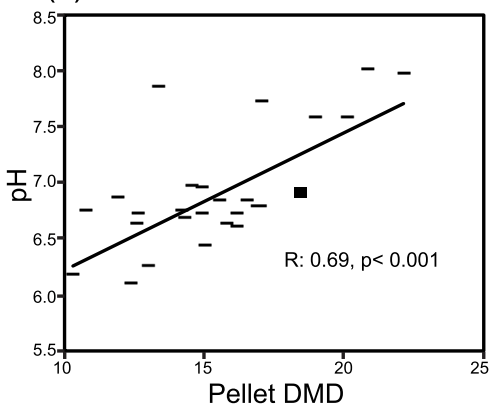

Firmicutes

Bacteroidetes

Spirochaetes

unclassified

Environmental parameter

- positive correlation $(p<0.05)$

- negative correlation $(p<0.05)$

으 $0.5-1 \%$

$10-15 \%$

$>30 \%$ 
Click here to download Supplementary Material: FEB28 Microbial Ecology TableS1.pdf 
Click here to download Supplementary Material: FEB28 Microbial Ecology TableS2.pdf 